Pacific Journal of Mathematic 


\title{
A BILATERAL GENERATING FUNCTION FOR THE ULTRASPHERICAL POLYNOMIALS
}

\author{
S. K. Chatterjea
}

The following differentiation formula for the Ultraspherical polynomials $P_{n}^{\lambda}(x)$ was given by Tricomi:

$$
P_{n}^{\lambda}\left(\frac{x}{\sqrt{x^{2}-1}}\right)=\frac{(-1)^{n}\left(x^{2}-1\right)^{\lambda+1 / 2 n}}{n !} D^{n}\left(x^{2}-1\right)^{-\lambda} .
$$

The object of this paper is to point out that the formula of Tricomi leads us to the following bilateral generating function for the Ultraspherical polynomials:

THEOREM.

then

$$
\text { If } F(x, t)=\sum_{m=0}^{\infty} a_{m} t^{m} P_{m}^{\lambda}(x),
$$

$$
\rho^{-2 \lambda} F\left(\frac{x-t}{\rho}, \frac{t y}{\rho}\right)=\sum_{r=v}^{\infty} t^{r} b_{r}(y) P_{r}^{\lambda}(x),
$$

where

$$
b_{r}(y)=\sum_{m=0}^{\infty}\left(\begin{array}{c}
r \\
m
\end{array}\right) a_{m} y^{m}, \quad \text { and } \quad \rho=\left(1-2 x t+t^{2}\right)^{1 / 2} .
$$

Starting from the formula (1.2), one can derive a large number of bilateral generating functions for the Ultraspherical polynomials by attributing different values to $a_{m}$.

2. Proof of the main formula (1.2). We first note from (1.1) that

$$
\left(\frac{1}{\sqrt{x^{2}-1}}\right)^{n} P_{n}^{\lambda}\left(\frac{x}{\sqrt{x^{2}-1}}\right)=\frac{(-1)^{n}}{n !}\left(x^{2}-1\right)^{\lambda} D^{n}\left(x^{2}-1\right)^{-\lambda} .
$$

Now let

$$
F\left(\frac{x}{\sqrt{x^{2}-1}}, \quad \frac{t}{\sqrt{x^{2}-1}}\right)=\sum_{m=0}^{\infty} a_{m}\left(\frac{t}{\sqrt{x^{2}-1}}\right)^{m} P_{m}^{\lambda}\left(\frac{x}{\sqrt{x^{2}-1}}\right)
$$

be a given generating function for $P_{n}^{\lambda}(x)$. Replacing $t$ by ty and multiplying both sides by $\left(x^{2}-1\right)^{-\lambda}$ and then operating $e^{-t D}$, we get

$$
\begin{aligned}
& e^{-t D}\left(x^{2}-1\right)^{-\lambda} F\left(\frac{x}{\sqrt{x^{2}-1}}, \frac{t y}{\sqrt{x^{2}-1}}\right) \\
& \quad=e^{-t D}\left(x^{2}-1\right)^{-\lambda} \sum_{m=0}^{\infty} a_{m}\left(\frac{t y}{\sqrt{x^{2}-1}}\right)^{m} P_{m}^{\lambda}\left(\frac{x}{\sqrt{x^{2}-1}}\right) .
\end{aligned}
$$

Since we know that 


$$
e^{-t D} f(x)=f(x-t),
$$

the left member of (2.2) is equal to

$$
\left\{(x-t)^{2}-1\right\}^{-\lambda} F\left(\frac{x-t}{\sqrt{(x-t)^{2}-1}}, \frac{t y}{\sqrt{(x-t)^{2}-1}}\right) \text {. }
$$

But the right member of (2.2) is equal to

$$
\begin{aligned}
\sum_{m=0}^{\infty} & a_{m}(t y)^{m} e^{-t D}\left(x^{2}-1\right)^{-\lambda}\left(\frac{1}{\sqrt{x^{2}-1}}\right)^{m} P_{m}^{\lambda}\left(\frac{x}{\sqrt{x^{2}-1}}\right) \\
& =\sum_{m=0}^{\infty} a_{m}(t y)^{m} e^{-t D}\left\{\frac{(-1)^{m}}{m !} D^{m}\left(x^{2}-1\right)^{-\lambda}\right\} \\
& =\sum_{m=0}^{\infty} a_{m}(t y)^{m} \sum_{r=0}^{\infty} \frac{(-t)^{r}}{r !} D^{r}\left\{\frac{(-1)^{m}}{m !} D^{m}\left(x^{2}-1\right)^{-\lambda}\right\} \\
& =\sum_{m=0}^{\infty} a_{m} y^{m} \sum_{r=0}^{\infty} \frac{(-t)^{r+m}}{r ! m !} D^{r+m}\left(x^{2}-1\right)^{-\lambda} \\
& =\left(x^{2}-1\right)^{-\lambda} \sum_{m=0}^{\infty} a_{m} y^{m} \sum_{r=0}^{\infty}\left({ }^{r+m}\right) t^{r+m}\left(\frac{1}{\sqrt{x^{2}-1}}\right)^{r+m} P_{r+m}^{\lambda}\left(\frac{x}{\sqrt{x^{2}-1}}\right) \\
& =\left(x^{2}-1\right)^{-\lambda} \sum_{r=0}^{\infty}\left(\frac{t}{\sqrt{x^{2}-1}}\right)^{r} P_{r}^{\lambda}\left(\frac{x}{\sqrt{x^{2}-1}}\right) \sum_{m=0}^{r}\left(\begin{array}{c}
r \\
m
\end{array}\right) a_{m} y^{m} \cdot
\end{aligned}
$$

It follows therefore that: If

$$
F\left(\frac{x}{\sqrt{x^{2}-1}}, \quad \frac{t}{\sqrt{x^{2}-1}}\right)=\sum_{m=0}^{\infty} a_{m}\left(\frac{t}{\sqrt{x^{2}-1}}\right)^{m} P_{m}^{\lambda}\left(\frac{x}{\sqrt{x^{2}-1}}\right),
$$

then

$$
\begin{aligned}
& \left\{\frac{(x-t)^{2}-1}{x^{2}-1}\right\}^{-\lambda} F\left(\frac{x-t}{\sqrt{(x-t)^{2}-1}}, \frac{t y}{\sqrt{(x-t)^{2}-1}}\right) \\
& =\sum_{r=0}^{\infty}\left(\frac{t}{\sqrt{x^{2}-1}}\right)^{r} b_{r}(y) P_{r}^{\lambda}\left(\frac{x}{\sqrt{x^{2}-1}}\right),
\end{aligned}
$$

where $b_{r}(y)=\sum_{m=0}^{r}\left(\begin{array}{c}r \\ m\end{array}\right) a_{m} y^{m}$. Now changing $x\left(x^{2}-1\right)^{-1 / 2}$ into $x$ and then $t$ into $t\left(x^{2}-1\right)^{-1 / 2}$, we obtain the theorem mentioned in the introduction.

3. Some applications of the theorem.

(A) First we consider the generating function of Truesdell:

$$
e^{x t}{ }_{\circ} F_{1}\left(-; \lambda+\frac{1}{2} ; \frac{t^{2}\left(x^{2}-1\right)}{4}\right)=\sum_{m=0}^{\infty} \frac{t^{m}}{(2 \lambda)_{m}} P_{m}^{\lambda}(x) \text {. }
$$

Thus if we take $a_{m}=1 /(2 \lambda)_{m}$ in our theorem, we obtain

$$
\rho^{-2} \exp \left\{\frac{y t(x-t)}{\rho^{2}}\right\}{ }_{\circ} F_{1}\left(-; \lambda+\frac{1}{2} ; \frac{y^{2} t^{2}\left(x^{2}-1\right)}{4 \rho^{4}}\right)=\sum_{r=0}^{\infty} t^{r} b_{r}(y) P_{r}^{\lambda}(x) \text {. }
$$


But we notice that

$$
b_{r}(y)={ }_{1} F_{1}(-r ; 2 \lambda ;-y)=\frac{r !}{(2 \lambda)_{r}} L_{r}^{(2 \lambda-1)}(-y) .
$$

Hence we derive the following generating function of Weisner [3].

$$
\begin{aligned}
\rho^{-2 \lambda} & \exp \left\{\frac{-y t(x-t)}{\rho^{2}}\right\}{ }_{\circ} F_{1}\left(-; \lambda+\frac{1}{2} ; \frac{y^{2} t^{2}\left(x^{2}-1\right)}{4 \rho^{4}}\right) \\
& =\sum_{r=0}^{\infty} \frac{r ! L_{r}^{(2 \lambda-1)}(y)}{(2 \lambda)_{r}} t^{r} P_{r}(x) .
\end{aligned}
$$

Thus we remark that the bilateral generating function of Weisner is a particular case of our theorem. Moreover we have obtained the theorem by a method different from that used by Weisner or from that used by Rainville [2].

(B) If we consider the formula of Brafman:

$$
\begin{aligned}
(1 & -x t)^{-\gamma}{ }_{2} F_{1}\left(\frac{1}{2} \gamma, \frac{1}{2} \gamma+\frac{1}{2} ; \lambda+\frac{1}{2} ; \frac{t^{2}\left(x^{2}-1\right)}{(1-x t)^{2}}\right) \\
& =\sum_{m=0}^{\infty} \frac{(\gamma)_{m} t^{m}}{(2 \lambda)_{m}} P_{m}^{\lambda}(x),
\end{aligned}
$$

then we put $a_{m}=(\gamma)_{m} /(2 \lambda)_{m}$ in our theorem and we obtain

$$
\begin{aligned}
& \rho^{2(\gamma-\lambda)}\left\{\rho^{2}+y t(x-t)\right\}^{-\gamma}{ }_{2} F_{1}\left(\frac{1}{2} \gamma, \frac{1}{2} \gamma+\frac{1}{2} ; \lambda+\frac{1}{2} ; \frac{y^{2} t^{2}\left(x^{2}-1\right)}{\left(\rho^{2}+y t(x-t)\right)^{2}}\right) \\
& \quad=\sum_{r=0}^{\infty}{ }_{2} F_{1}(-r, \gamma ; 2 \lambda ; y) t^{r} P_{r}^{\lambda}(x) .
\end{aligned}
$$

(C) Next we consider the following generating function of Bateman:

$$
\begin{aligned}
& { }_{0} F_{1}\left(-; \lambda+\frac{1}{2} ; \frac{t(x-1)}{2}\right)_{0} F_{1}\left(-; \lambda+\frac{1}{2} ; \frac{t(x+1)}{2}\right) \\
& \quad=\sum_{m=0}^{\infty} \frac{t^{m}}{(2 \lambda)_{m}\left(\lambda+\frac{1}{2}\right)_{m}} P_{m}^{\lambda}(x) .
\end{aligned}
$$

Here we set $a_{m}=1 /\left\{(2 \lambda)_{m}(\lambda+1 / 2)_{m}\right\}$ in our theorem and we derive

$$
\begin{aligned}
& \rho^{-2 \lambda}{ }_{0} F_{1}\left(-; \lambda+\frac{1}{2} ; \frac{y t(t-x+\rho)}{2 \rho^{2}}\right)_{0} F_{1}\left(-; \lambda+\frac{1}{2} ; \frac{y t(t-x-\rho)}{2 \rho^{2}}\right) \\
& \quad=\sum_{r=1}^{\infty}{ }_{1} F_{2}\left(-r ; 2 \lambda, \lambda+\frac{1}{2} ; y\right) t^{r} P_{r}^{\lambda}(x) .
\end{aligned}
$$

(D) Lastly if we consider the following generating function of Brafman: 
(3.7)

$$
\begin{gathered}
{ }_{2} F_{1}\left(\gamma, 2 \lambda-\gamma ; \lambda+\frac{1}{2} ; \frac{1-t-\rho}{2}\right) x \\
{ }_{2} F_{1}\left(\gamma, 2 \lambda-\gamma ; \lambda+\frac{1}{2} ; \frac{1+t-\rho}{2}\right) \\
\quad=\sum_{m=0}^{\infty} \frac{(\gamma)_{m}(2 \lambda-\gamma)_{m}}{(2 \lambda)_{m}\left(\lambda+\frac{1}{2}\right)_{m}} t^{m} P_{m}^{\lambda}(x) ;
\end{gathered}
$$

we put

$$
a_{m}=\frac{(\gamma)_{m}(2 \lambda-\gamma)_{m}}{(2 \lambda)_{m}\left(\lambda+\frac{1}{2}\right)_{m}}
$$

in our theorem and thus we obtain

$$
\begin{aligned}
& \rho^{-2 \lambda}{ }_{2} F_{1}\left(\gamma, 2 \lambda-\gamma ; \lambda+\frac{1}{2} ; \frac{\rho+y t-\omega}{2 \rho}\right) x \\
& { }_{2} F_{1}\left(\gamma, 2 \lambda-\gamma ; \lambda+\frac{1}{2} ; \frac{\rho-y t-\omega}{2 \rho}\right) \\
& \quad=\sum_{r=0}^{\infty}{ }_{3} F_{2}\left(-r, \gamma, 2 \lambda-\gamma ; 2 \lambda, \lambda+\frac{1}{2} ; y\right) t^{r} P_{r}^{\lambda}(x) ;
\end{aligned}
$$

where

$$
\omega=\left[1-2 x t(1-y)+t^{2}(1-y)^{2}\right]^{1 / 2} .
$$

\section{REFERENCES}

1. A. Erdelyi, et al., Higher transcendental functions, MeGraw Hill Book Co., New York, 1953.

2. E. D. Rainville, Special functions, Macmillan Co., New York, 1960.

3. L. Weisner, Group-theoretic origins of certain generating functions, Pacific J. Math. 5, (1955), 1033-1039.

Received April 20, 1968.

Bangabasi College

Calcutta 



\section{Pacific Journal of Mathematics \\ Vol. 29, No. 1 \\ May, 1969}

Jorge Alvarez de Araya, A Radon-Nikodým theorem for vector and operator

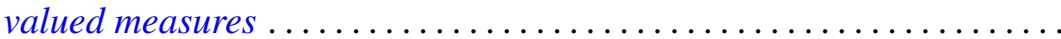

Deane Eugene Arganbright, The power-commutator structure of finite

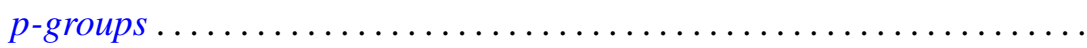

Richard Eugene Barlow, Albert W. Marshall and Frank Proschan, Some

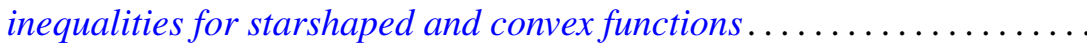

David Clarence Barnes, Some isoperimetric inequalities for the eigenvalues

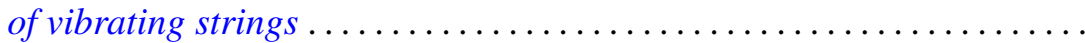

David Hilding Carlson, Critical points on rim-compact spaces ...........

Allan Matlock Weber Carstens, The lattice of pretopologies on an arbitrary

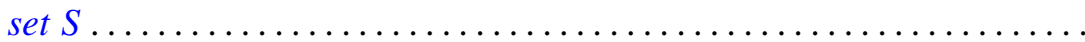

S. K. Chatterjea, A bilateral generating function for the ultraspherical

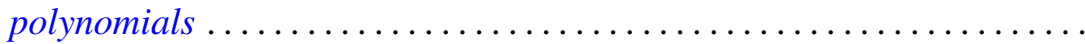

Ronald J. Ensey, Primary Abelian groups modulo finite groups ......... 77

Harley M. Flanders, Relations on minimal hypersurfaces ............ 83

Allen Roy Freedman, On asymptotic density in n-dimensions........... 95

Kent Ralph Fuller, On indecomposable injectives over artinian rings...... 115

George Isaac Glauberman, Normalizers of p-subgroups in finite groups . . . 137

William James Heinzer, On Krull overrings of an affine ring ........... 145

John McCormick Irwin and Takashi Ito, A quasi-decomposable abelian group without proper isomorphic quotient groups and proper isomorphic subgroups.

Allan Morton Krall, Boundary value problems with interior point boundary conditions

John S. Lowndes, Triple series equations involving Laguerre

polynomials

Philip Olin, Indefinability in the arithmetic isolic integers

Ki-Choul Oum, Bounds for the number of deficient values of entire functions whose zeros have angular densities..

R. D. Schafer, Standard algebras ....................

Wolfgang M. Schmidt, Irregularities of distribution. III.

Richard Alfred Tapia, An application of a Newton-like method to the Euler-Lagrange equation 